\title{
Vector Addition in Different Contexts
}

\author{
Philip Southey* and Saalih Allie*đI \\ *Department of Physics, University of Cape Town, Rondebosch, South Africa, 7708

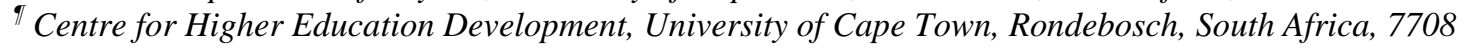

\begin{abstract}
The acquisition metaphor of learning is often used by teachers of physics: Students are thought to acquire a particular concept and then transfer this concept to new contexts. For example, one might argue that students acquire the mathematical concept of "vector addition" and then apply it in different physical contexts. In this study, 200 freshmen taking an introductory physics course were asked to calculate total force, total displacement and total momentum in simple contexts involving vector addition at right angles. Another similar group of students were asked to calculate net force, net displacement, and net momentum. When asked to calculate the "net" quantity, students did significantly better than when asked to calculate the "total" quantity. Students did significantly worse when adding momenta as opposed to adding forces or displacements. These results are inconsistent with a basic "acquisition-transfer" perspective of learning. An analysis of subsequent interviews and questionnaires was also conducted.
\end{abstract}

Keywords: Vectors, Mechanics, Context

PACS: 01.40.Fk

\section{INTRODUCTION}

Physics education practice and research are guided by overarching theoretical frameworks. One of the more important frameworks is that of conceptual change. In his paper "A History of Conceptual Change" diSessa [1] argues that a major fault line in the conceptual change literature is that of coherence/fragmentation: to what extent are student ideas coherent and integrated, or quasi-independent and fragmented. Toward one end of this axis is the "theory theory": the idea that students' concepts cohere in robust (albeit naïve) theories. Toward the other end of the axis is the "knowledge in pieces" framework developed by diSessa and Hammer among others. As the names suggest, the theory theory is more compatible with notions of abstraction and transfer, while the knowledge in pieces framework foregrounds the role of context. Indeed, diSessa argues that future research into conceptual change ought to make contextuality a central concern.

Previous research has demonstrated that students' understanding of vectors is sensitive to various contexts. Shaffer and McDermott [2] showed that students were better able to subtract vectors when no physical context was involved. When discussing implications for developing tutorial curricula, they cite context as being 'critically important'. In a paper which presents his cumulative knowledge of teaching vectors to students, Roche [3] observes that "the displacement vector is easiest to explain, perhaps too easy. It is very different from other vectors such as force." Barniol and Zavala [4] show that student responses do indeed exhibit context sensitivity depending on whether the vectors to be added are displacements or forces. Barniol demonstrates this sensitivity in a graphical context (students are given two arrows drawn on graph paper and required to draw the resultant vector) while this paper describes an algebraic context (see below). Allie and Buffler [5] also found that students were sensitive to the particular vector quantity being added. The present study aims to explore these findings in more detail.

\section{METHODOLOGY I}

An exploratory multiple choice questionnaire was administered in February 2013. The first question asked students to categorize various physical quantities (force, displacement and momentum) as being vectors or scalars. The remaining three questions involved quantitative vector addition. Students were able to select a number from $0-11$ as their answer. For the sake of brevity, these multiple choice answers are only included for question 4 below. The following instructions appeared at the top of the sheet:

In each of the problems below circle the answer that you think is correct. Where the answer is a vector you need only select the magnitude.

2. Two forces are applied to a box. Force 1 has a magnitude of 3 Newtons and acts East while force 2 has a magnitude of $4 N$ and acts North. The total force on the box is:

3. A student walks $2 \mathrm{~m}$ West, then $5 \mathrm{~m}$ East and finally $4 \mathrm{~m}$ North. The final displacement of student is:

4. Ball 1 has a mass of $1.5 \mathrm{~kg}$ and a velocity of $2 \mathrm{~ms}^{-1}$ North while ball 2 has a mass of $1 \mathrm{~kg}$ and a velocity of $4 \mathrm{~ms}^{-1}$ East. The total momentum of the two balls is: 


$\begin{array}{lllllll} & 0 & 1 & 2 & 3 & 4 & 5\end{array}$

Note that questions 2, 3 and 4 all require the same calculation of the hypotenuse of a 3-4-5 triangle to give a correct answer of 5 .

\section{Sample}

The sample of students comprised 227 freshmen enrolled for a medical degree at the University of Cape Town (UCT). The students would have encountered two dimensional addition of forces and displacements at high school, but would have been exposed to adding momenta in only one dimension. The questionnaire was given at the beginning of the university year, prior to instruction.

\section{FINDINGS}

The vast majority of students correctly identified force, displacement and momentum as vector quantities (94\%, 93\% and $88 \%$ respectively). However, they were not as successful when adding these various quantities.

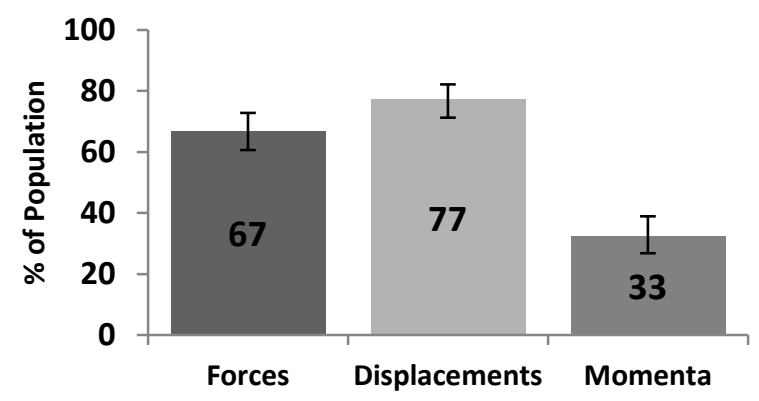

FIGURE 1. Percentage of students to correctly add vectors in different contexts $(n=227)$.

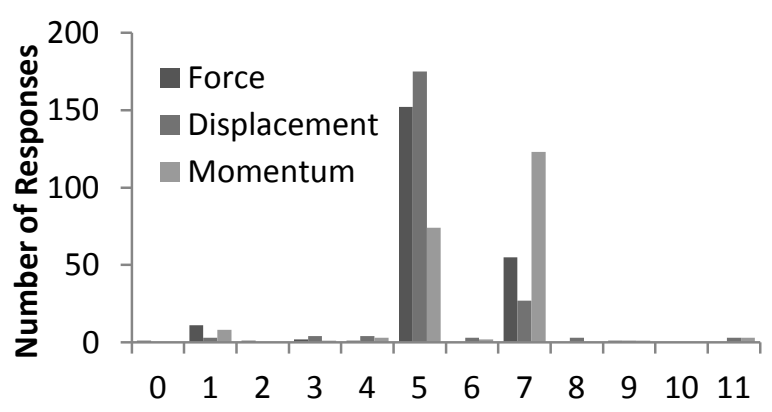

FIGURE 2. Distribution of answers for the addition of various vector quantities $(n=227)$.

Figure 1 shows the results for questions $2-4$. Figure 2 shows that students' responses clustered around the multiple choice values of 5 and 7 .

\section{METHODOLOGY II}

\section{Follow up Interviews}

Follow up interviews were conducted with eight students. These students were selected for having drawn the correct vector diagram (i.e. a 3-4-5 triangle) on their answer sheets but choosing the incorrect answer of $7 \mathrm{~N}$ and/or $7 \mathrm{kgms}^{-1}$. The interviews occurred 8 months after they had answered the questionnaire during which time they had completed one semester of physics 1 instruction. The students were given their previous answer sheet, asked to look over their previous answers, and to explain why they might agree or disagree with any of answers they had given.

\section{ANALYSIS}

The interview data was analyzed using an approach suggested by Grounded Theory. General patterns were identified within students' responses. Three of these general patterns are described below.

\section{FINDINGS}

1. Adding momenta is different to adding forces because momentum is different to force.

Students often cited their lack of knowledge of momentum as a reason for their difficulty with adding momenta. However, from an expert's point of view, they had all the relevant information they required, as they had all correctly indicated that momentum was a vector quantity. Three of the students acknowledged that they had initially seen the force question as being similar to the momentum question, but that some aspect of the question had subsequently led them to believe that different addition strategies were required: "...basically these two questions [q2 and q4] are the same, like to me they look the same... but when I see total momentum and I see total force, my thinking changes."

\section{Students have different conceptions of "total"} and "net".

Four of the students explicitly identified a difference between a total vector quantity and a net or resultant vector quantity. On trying to disentangle the notions of "total" and "net" some students gave a procedural distinction: "whenever I hear resultant I just automatically go for the calculation where you use the triangle..." Other students provided a more robust conceptual distinction: "I do agree that there is 7 newtons applied to the box, but, it's a 5 newton... result. ... Just think, if I was getting punched with 3 
newtons and 4 newtons [gestures throwing punches] I'd have 7 newtons pressed on me... in total." Some students claimed that it was the word "total" that caused them to sum the magnitudes algebraically and that they would have answered differently if they had been asked for the net quantity.

3. The notion of a system: students have robust ideas of separateness and togetherness

While correctly revising their answers for the momentum question, three students changed their minds and reverted back to their incorrect answer because of the notion of "separateness":

"...I think what I did was took momentum as a scalar quantity and just added the two... whereas I think what should happen is... [inaudible]... no...I think its fine...because the two balls are unrelated and moving in different directions... you can't sort of have the resultant momentum between the two."

When prompted, all students admitted that if the question had read: "Find the momentum of the two ball system" instead of "Find the momentum of the two balls", they would have given the correct answer of $5 \mathrm{kgms}^{-1}$.

\section{METHODOLOGY III}

\section{Questionnaire 2}

A revised questionnaire was given to a new cohort of pre-medical freshmen in February 2014. Approximately half of the class received the identical multiple choice questions as the 2013 cohort. The other half received similar questions, but with three small changes: Instead of being asked for the total force, total momentum and total displacement, they were asked for the net force, net momentum and net displacement. Thereafter, all students were given the following scenario:

As in question 1 above: Two forces are applied to a box. Force 1 has a magnitude of $3 N$ and acts East while force 2 has a magnitude of $4 \mathrm{~N}$ and acts North. Three students have the following conversation:

Student A: "The magnitude of the total force on the box is $7 \mathrm{~N}$, and the magnitude of the net force on the box is $7 \mathrm{~N}$."

Student B: "I agree that the magnitude of the total force on the box is $7 \mathrm{~N}$, but the magnitude of the net force on the box is $5 \mathrm{~N}$."

Student C: "The magnitude of the total force on the box is $5 \mathrm{~N}$ and the magnitude of the net force on the box is $5 \mathrm{~N}$."

(i) With whom do you most closely agree? (Circle $\begin{array}{llll}\text { your choice). } \quad A & B\end{array}$

(ii) Explain why you agree with that student:
Note: If you would like to change your answer to question 1 on page 1, please indicate here what answer you would now choose. (Do not go back and change your answer).

They were then given a similar scenario where three posited students are discussing possible values for total momentum and net momentum.

\section{FINDINGS}

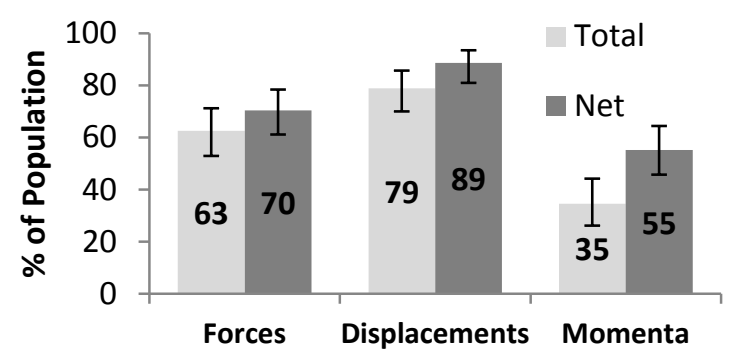

FIGURE 3. Percentage of students to correctly add vectors in different contexts.

The light grey columns in Figure 3 represent the proportion of correct answers for "total force", "total displacement" and "total momentum". The dark grey columns are the proportion of correct answers for the equivalent net vector quantities. As in Figure 1, Figure 3 demonstrates that students perform differently depending on the type of vector quantity being added.

As indicated above, students were given the opportunity to indicate a desire to change their answers once they had encountered the discussion regarding the possible differences between "total" and "net" vector quantities. These adjusted values are given below:

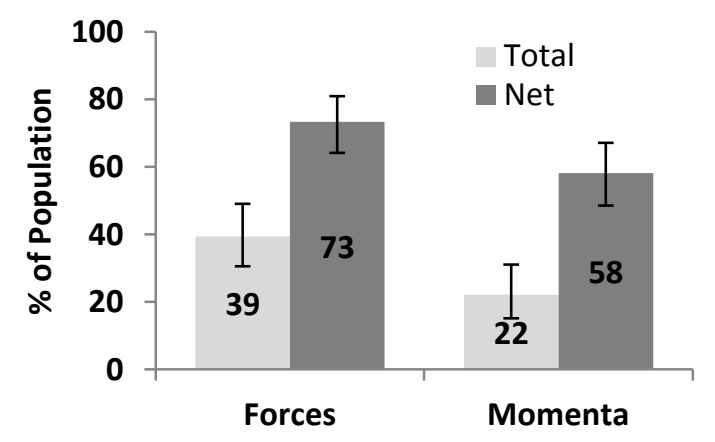

FIGURE 4. Percentage of students to correctly add vectors in different contexts, once exposed to the idea that a "total vector quantity" might differ from a "net vector quantity".

Figure 4 shows us that after exposure to the idea that the total force (total momentum) might be different to net force (net momentum) a large number of students adjusted their previously correct result of $5 \mathrm{~N}$ for total force $\left(5 \mathrm{~kg} \mathrm{~ms}^{-1}\right.$ for total momentum) to the incorrect 
value of $7 \mathrm{~N}\left(7 \mathrm{kgms}^{-1}\right)$. A far smaller number of students requested their previously incorrect value of " 7 " for the net value to be changed to the correct value of " 5 ".

Perhaps the most interesting result was students' overwhelming support for a clear distinction between total vector and net vector. $82 \%$ of students thought that "total force" and "net force" were different with total force $=7 \mathrm{~N}$ and net force $=5 \mathrm{~N}$, while $60 \%$ of students thought that total momentum and net momentum were different. Only $12 \%$ (10\%) correctly identified total force (momentum) and net force (momentum) as being synonymous, and having a value of $5 \mathrm{~N}\left(5 \mathrm{kgms}^{-1}\right)$. The remaining $6 \%$ (30\%) of students thought that total force (momentum) and net force (momentum) both had a value of $7 \mathrm{~N}\left(7 \mathrm{kgms}^{-1}\right)$.

\section{DISCUSSION}

If one adopts the "theory theory" framework of conceptual change, one may assume that students acquire a robust, coherent conception of vector addition after successfully adding vectors in one particular context. If they identify other physical quantities as being vectors, it would be reasonable to assume that they could transfer their knowledge of vector addition from one context to another. However, Figure 1 demonstrates that even though students correctly identified force, displacement and momentum as vector quantities, their success at vector addition is sensitive to the context of the particular vector quantity being added ( $p<0.001$, Cochran's $q$ test). Figure 4 demonstrates that students are also sensitive to whether they are asked for the total or net quantity, if it is suggested that "total vector quantity" may differ from "net vector quantity" ( $\mathrm{p}<0.001$ for both cases; two chi squared tests with Bonferoni correction, $\alpha=0.005)$. The particular sensitivity to momentum (see the third column of Figure 3) might further support the hypothesis that when students encounter new material, they are particular sensitive to textual cues which an expert would ignore.

Introductory textbooks ordinarily do not bring attention to the fact that "total" and "net" are synonymous in the context of vector addition. While most first year textbooks use "resultant force", there are examples of first year textbooks, such as Reese's "University Physics" [6], that use "total force" throughout.

Finally, it is worth re-iterating that this sensitivity to context (of the particular vector quantity being added) appears both in a graphical context [4], where a continuum of answers are available (i.e. the resultant vector could assume any possible length), and in an algebraic context, where a discrete number of answers are available (see Figure 2). The "dual appearance" of this context sensitivity should influence further discussion regarding the potential cognitive modeling of this particular sensitivity to context.

A possible avenue of further research might be investigating students' naïve conceptions of total force. Our interview data suggest that students may have distinct notions of the 'total felt effect' (e.g. experiencing a 'total punch' of seven newtons) and the 'resultant motion effect', which may map onto naïve conceptions of 'total force' and 'net force' respectively.

\section{CONCLUSION}

The data presented here present a strong case that when students perform the algebraic, two dimensional addition of vector quantities they are sensitive both to (a) the type of vector quantity being added (force, displacement, momentum) and (b) to the textual prompts of "total" and "net". Furthermore, the latter sensitivity is particularly apparent if the students are presented with (i) a vector quantity with which they are not very familiar (i.e. momentum) or (ii) if it is suggested that there might be a difference between the notions of "total" and "net". The data suggest that students may not have a robust, coherent conception of vector addition, and therefore support a knowledge in pieces perspective of conceptual change.

\section{ACKNOWLEDGEMENTS}

Thank you to SASOL Inzalo Foundation, Dr. Peterson, and members of UCT Physics Department.

\section{REFERENCES}

[1] A. DiSessa, "A History of Conceptual Change Research: Threads and Fault Lines.," in The Cambridge Handbook of the Learning Sciences, R. K. Sawyer, Ed. Cambridge University Press, 2006, pp. 265-281.

[2] P. S. Shaffer and L. C. McDermott, "A researchbased approach to improving student understanding of the vector nature of kinematical concepts," Am. J. Phys., vol. 73, no. 10, pp. 921-931, 2005.

[3] J. Roche, "Introducing Vectors.," Phys. Educ., vol. 32, no. 5, pp. 339-345, 1997.

[4] P. Barniol and G. Zavala, "Vector addition: Effect of the context and position of the vectors," in AIP Conference Proceedings, 2010, vol. 1289, pp. 7376.

[5] S. Allie and A. Buffler, "A course in tools and procedures for Physics I," Am. J. Phys., vol. 66, p. 613, 1998

[6] R. L. Reese, University physics, no. v. 1. Brooks/Cole Pub. Co., 2000. 